\title{
Research utility of noninvasive methods for measurement of cardiac output
}

\begin{abstract}
Two noninvasive methods of cardiac index $(Q)$ determination, pulsed Doppler echocardiography $\left(Q_{\text {Dop }}\right)$, and $\mathrm{CO}_{2}$ rebreathing $\left(\mathrm{Q}_{\mathrm{co}_{2}}\right)$ were compared to dye dilution in eight normal volunteers. Measurements of $\mathrm{Q}$ were made by dye dilution $\left(\mathrm{Q}_{\mathrm{DD}}\right)$ and the two noninvasive techniques under the following conditions: (1) supine rest, (2) after inflation of cuffs around the thighs, (3) 35-degree head-up tilt, (4) supine rest repeated, (5) during constant isoproterenol infusion, and (6) after an intravenous bolus of propranolol. When mean $Q$ values of the group for each intervention were compared, close agreement was observed between dye dilution and each noninvasive method $\left(Q_{D O P}=1.08 Q_{D D}-0.07 \mathrm{~L} / \mathrm{min} \cdot \mathrm{m}^{2}, r=0.99\right.$, $\left.\mathrm{SEE}=0.02 ; \mathrm{Q}_{\mathrm{CO}_{2}}=0.68 \mathrm{QDD}_{\mathrm{DD}}+0.84 \mathrm{~L} / \mathrm{min} \cdot \mathrm{m}^{2}, \mathrm{r}=0.97, \mathrm{SEE}=0.02\right)$. Fair correlations were achieved when all 48 paired observations were analyzed $\left(Q_{D o p}=1.00 Q_{D D}+0.17 \mathrm{~L} / \mathrm{min} \cdot \mathrm{m}^{2}, r=0.89\right.$, $\left.\mathrm{SEE}=0.17 ; \mathrm{Q}_{\mathrm{CO}_{2}}=0.71 \mathrm{Q}_{\mathrm{DD}}+0.77 \mathrm{~L} / \mathrm{min} \cdot \mathrm{m}^{2}, \mathrm{r}=0.79, \mathrm{SEE}=0.20\right)$. However, the Doppler ultrasound technique was significantly more precise in quantitating changes in $Q$ in individual patients. These results demonstrate that estimates of $\mathrm{Q}$ can be made with reasonable accuracy by either $\mathrm{CO}_{2}$ rebreathing or Doppler echocardiography. However, the Doppler technique is a much more accurate means of quantitating acute changes in Q. (Clin Pharmacol Ther 1987;41:419-25.)
\end{abstract}

\author{
Alan L. Hinderliter, M.D., M. Andrew Fitzpatrick, M.D., Nicholas Schork, B.S., \\ and Stevo Julius, M.D., Sc.D. Ann Arbor, Mich.
}

Much progress in our understanding of the physiology of the cardiovascular system and the pathophysiology of its disorders has stemmed from hemodynamic research in human subjects. The accurate determination of cardiac output $(\mathrm{Q})$ has been essential for such studies. Until recently, however, the most reliable methods of $\mathrm{Q}$ measurement have required right heart catheterization and arterial cannulation. These invasive techniques are associated with some discomfort and morbidity and are not well suited for repeated measures over periods longer than several hours.

Recent technologic advances in Doppler echocardiography have made possible accurate noninvasive estimates of Q. A number of investigators have demonstrated that $\mathrm{Q}$ calculated from Doppler measurements of ascending aortic blood flow in resting patients correlates well with values obtained by thermodilution. ${ }^{1-5}$

From the Division of Hypertension, Department of Internal Medicine, University of Michigan Medical Center.

Supported by the National Institutes of Health grant no. HL-21893 and Merck Sharp \& Dohme.

Received for publication April 8, 1986; accepted Sept. 8, 1986. Reprint requests: Stevo Julius, M.D., Sc.D., Division of Hypertension, 3918 Taubman Center, University of Michigan Medical Center, Ann Arbor, MI 49109-0356.
Others have shown the usefulness of the Doppler technique in estimating $Q$ changes in response to vasodilators and inotropic agents. ${ }^{6-8}$

This study was undertaken to further evaluate the ability of Doppler ultrasonography to measure serial changes in $\mathrm{Q}$ in human laboratory subjects. In addition, we compared the accuracy of this technique to that of $\mathrm{CO}_{2}$ rebreathing, a noninvasive means of $\mathrm{Q}$ measurement used previously in our laboratory. In contrast to the Fick and dye-dilution methods, in which $Q$ is measured directly, Doppler ultrasonography measures stroke volume (SV); Q is then calculated as a function of heart rate (HR). Validation of the Doppler method then requires demonstration that it is capable of quantitating changes in $\mathrm{Q}$ that are independent of $\mathrm{HR}$ alterations. For this reason our study was performed in subjects who underwent a series of maneuvers designed to produce a variety of $Q$ and HR changes. Specifically, with isoproterenol we increased both Q and HR; with tilt we decreased Q but increased HR; with propranolol both Q and HR were decreased; and finally with thigh cuff inflation we decreased $Q$ without altering HR. After each intervention, $Q$ was measured by both noninvasive techniques and the results were compared to dye-dilution determinations. 
Table I. HR and Q as measured by dye dilution, Doppler, and $\mathrm{CO}_{2}$ rebreathing with each intervention

\begin{tabular}{lcccc}
\hline Intervention & $\begin{array}{c}H R \\
(\mathrm{bpm})\end{array}$ & $\begin{array}{c}\text { Dye dilution } \\
\left(\mathrm{L} / \mathrm{min} \cdot \mathrm{m}^{2}\right)\end{array}$ & $\begin{array}{c}\text { Doppler } \\
\left(\mathrm{L} / \mathrm{min} \cdot \mathrm{m}^{2}\right)\end{array}$ & $\begin{array}{c}\mathrm{CO}_{2} \text { rebreathing } \\
\left(\mathrm{L} / \mathrm{min} \cdot \mathrm{m}^{2}\right)\end{array}$ \\
\hline Control 1 & $57.7 \pm 1.7$ & $3.04 \pm 0.40$ & $3.30 \pm 0.56$ & $2.81 \pm 0.64$ \\
Cuff & $58.3 \pm 2.1$ & $2.68 \pm 0.32$ & $2.87 \pm 0.45$ & $2.52 \pm 0.36$ \\
Tilt & $73.8 \pm 1.6$ & $2.45 \pm 0.34$ & $2.35 \pm 0.33$ & $2.74 \pm 0.52$ \\
Control 2 & $55.0 \pm 1.6$ & $2.93 \pm 0.45$ & $3.08 \pm 0.47$ & $2.74 \pm 0.54$ \\
Isoproterenol & $64.9 \pm 1.4$ & $4.52 \pm 0.65$ & $4.75 \pm 0.80$ & $3.95 \pm 0.81$ \\
Propranolol & $50.9 \pm 2.2$ & $2.49 \pm 0.38$ & $2.69 \pm 0.37$ & $2.51 \pm 0.50$ \\
\hline
\end{tabular}

Results are expressed as mean $=\mathrm{SE}$.

\section{METHODS}

Study group. The study group consisted of eight normal male volunteers ranging in age from 22 to 30 years. All had normal physical examinations, and none had any history of pulmonary or cardiovascular disease. Each subject read and signed a detailed informed consent form.

Protocol. The study protocol was approved by the Human Use Committee at the University of Michigan. After introduction to the laboratory, subjects were placed supine on a tilt table and catheters were inserted. Pressure cuffs were applied loosely around the thighs and, when required, pressured by a rapid inflator (D. E. Hokanson, Inc., Issaquah, Wash.).

Measurements of $\mathrm{Q}$ were then made by dye dilution, Doppler ultrasonography, and $\mathrm{CO}_{2}$ rebreathing under the following conditions: control 1: 15 minutes after insertion of catheters; cuff: 10 minutes after inflation of thigh cuffs to $60 \mathrm{~mm} \mathrm{Hg}$; tilt: 10 minutes after deflation of thigh cuffs and after assuming a 35-degree head-up tilt position; control 2: 10 minutes after returning to supine position; isoproterenol: during a constant intravenous infusion of isoproterenol titrated to produce an increase in HR of about $10 \mathrm{bpm}$ (10 to 15 $\mathrm{mEq} / \mathrm{kg} / \mathrm{min}$ in most cases); and propranolol: $30 \mathrm{~min}$ utes after administration of $0.2 \mathrm{mg} / \mathrm{kg}$ propranolol intravenously.

Doppler and rebreathing recordings were made by a single operator who was unaware of the dye-dilution results. Similarly, one investigator performed all of the dye-dilution measurements using a standard technique. Each dye dilution, Doppler, and rebreathing tracing was assigned a random number and interpreted blindly. All measurements were made in triplicate by each of the three methods and mean values were calculated.

Doppler measurements. A range-gated, pulsed Doppler system (ATL MK600 Adaptive Doppler Ultrasound; Advanced Technology Laboratories, Inc.,
Belleville, Wash.) was used to measure Q. Measurements of blood flow velocity in the ascending aorta were made with a small $3 \mathrm{MHz}$ transducer positioned in the suprasternal notch. With the exception of small adjustments made during head-up tilt, the same sampling depth was used for each set of observations in an individual subject. Analysis of returning Doppler signals was performed by the instrument every $10 \mathrm{msec}$ by fast Fourier transform, and the data were plotted with time along the $x$ axis, frequency shift along the $y$ axis, and the prevalence of frequency components in shades of gray. With the assumption that blood flow was parallel to the ultrasound beam, instantaneous velocity was calculated from the Doppler equation: $\mathrm{V}=\mathrm{cf}_{\mathrm{d}} / 2 \mathrm{f}_{\mathrm{o}}$, where $\mathrm{c}=$ the speed of sound in tissue $(1540 \mathrm{~cm} / \mathrm{sec}), f_{d}=$ Doppler frequency shift, and $\mathrm{f}_{0}=$ transmitted ultrasound frequency $(3 \mathrm{MHz})$.

Spectral images were recorded on dry silver paper and were analyzed by a method similar to that described by Elkayam et al. ${ }^{7}$ The flow-velocity integral (FVI), or the area under a flow-velocity curve, was estimated by the equation FVI $=0.57$ (PFV) (ET) $+0.9 \mathrm{~cm}$. This relation was derived by regression analysis of planimetered areas as a function of the products of PFV and ET in a separate series of curves $(r=0.98)$. The average FVI of five consecutive beats was calculated for each tracing.

The cross-sectional area (CSA) of the aorta was calculated from the diameter (D) as $\mathrm{CSA}=\pi \mathrm{D}^{2} / 4$. The diameter was measured from a standard 2-D longaxis parasternal view at a point just distal to the sinus of Valsalva. Images were frozen during systole (on the peak of the $\mathrm{T}$ wave), and measurements were made with electronic calipers using an inner-to-inner wall convention. The CSA used in calculating $Q$ values for each patient was the arithmetic mean of three separate determinations. It was assumed that the dimensions of the ascending aorta remained constant through- 
Table II. Correlation coefficients for each patient relating $Q$ values measured by dye dilution to values measured by Doppler and $\mathrm{CO}_{2}$ rebreathing

\begin{tabular}{lcc}
\hline Patient & Doppler & $\begin{array}{c}\mathrm{CO}_{2} \\
\text { rebreathing }\end{array}$ \\
\hline P. P. & 0.99 & 0.94 \\
D. L. & 0.97 & 0.95 \\
J. G. & 0.96 & 0.80 \\
D. S. & 0.98 & 0.97 \\
R. Q. & 0.91 & 0.86 \\
J. S. & 0.98 & 0.81 \\
D. W. & 0.87 & 0.84 \\
P. H. & 0.99 & 0.92 \\
Mean & 0.96 & 0.89 \\
\hline
\end{tabular}

out the study (i.e., that they were not changed by the interventions used to alter hemodynamics).

HR was determined from a simultaneously recorded ECG.

$\mathrm{Q}$ was calculated as the product of FVI, CSA, and HR divided by body surface area (BSA).

$\mathrm{CO}_{2}$ rebreathing measurements. Q measured by the rebreathing technique was calculated from the following equation:

$$
\mathrm{Q}=\left(\dot{\mathrm{V}}_{\mathrm{CO}_{2}} /\left[\mathrm{C}_{\mathrm{CO}_{2}-\mathrm{CACO}_{2}}\right]\right) / \mathrm{BSA}
$$

where $\mathrm{Q}=$ cardiac output in liters per minute, $\dot{\mathrm{V}}_{\mathrm{CO}_{2}}=$ $\mathrm{CO}_{2}$ production in milliliters per minute $\mathrm{STP}, \mathrm{C} \overline{\mathrm{v}}_{\mathrm{CO}_{2}}=$ $\mathrm{CO}_{2}$ content of mixed venous blood in milliliters per liter, and $\mathrm{Ca}_{\mathrm{CO}_{2}}=\mathrm{CO}_{2}$ content of arterial blood in milliliters per liter.

Instantaneous determinations of $\mathrm{PCO}_{2}$ were made with a Hewlett-Packard Model 47210A Capnometer (Hewlett-Packard Co., Medical Products, Waltham, Mass.) connected in series with the mouthpiece of the rebreathing apparatus.

$\dot{\mathrm{V}}_{\mathrm{CO}_{2}}$ was calculated as the product of minute ventilation, determined by a timed collection, and the mean concentration of $\mathrm{CO}_{2}$ in expired gas.

$\mathrm{C} \overline{\mathrm{V}}_{\mathrm{CO}_{2}}$ was measured indirectly by the equilibration rebreathing method initially described by Collier et al. ${ }^{9}$

$\mathrm{Ca}_{\mathrm{CO}_{2}}$ was derived from the $\mathrm{PCO}_{2}$ measured at endtidal expiration.

A standard $\mathrm{CO}_{2}$ dissociation curve for oxygenated blood was used to convert partial pressures to $\mathrm{CO}_{2}$ content. ${ }^{10}$

Studies were considered acceptable if end-tidal $\mathrm{PCO}_{2}$ varied by $1 \mathrm{~mm} \mathrm{Hg}$ on the three breaths preceding the rebreathing procedure and if a "plateau" was achieved within 15 seconds from the start of rebreathing.
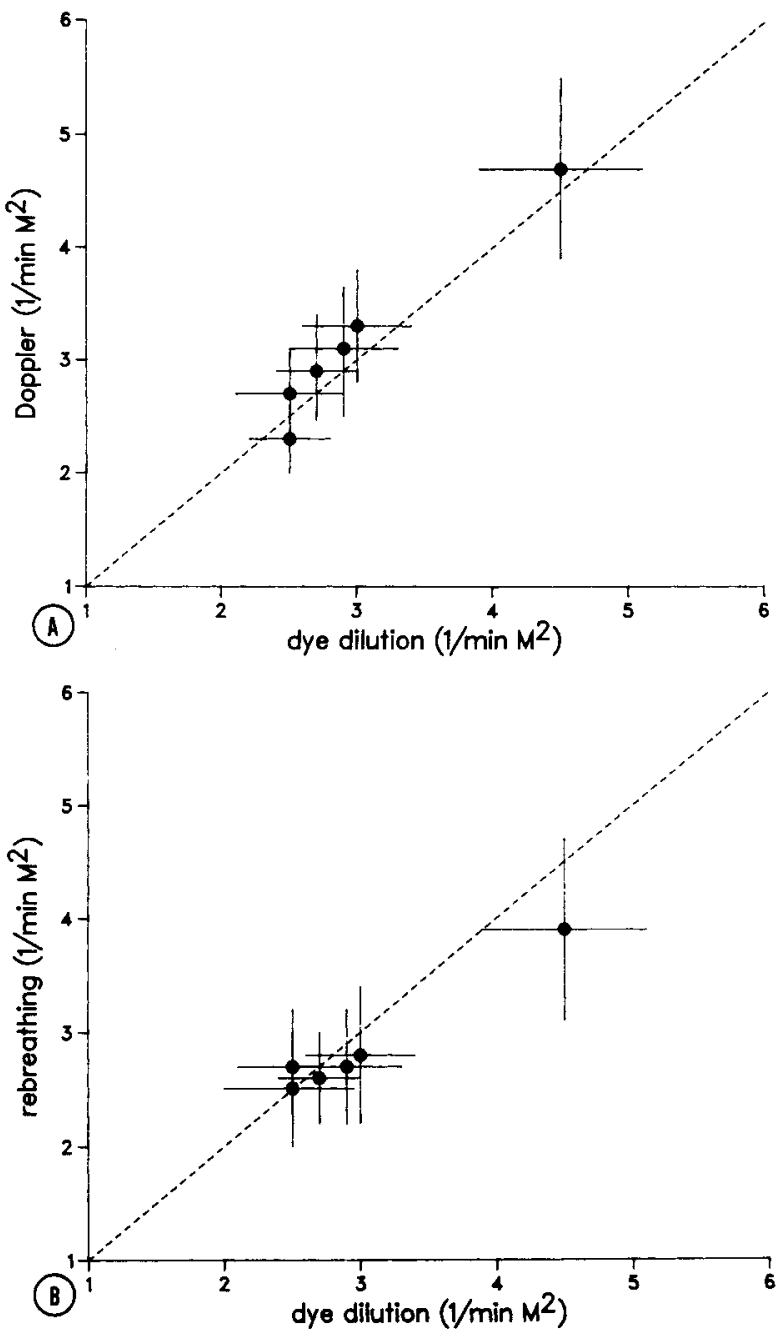

Fig. 1. Relationship between mean $Q$ values during each intervention as measured by dye dilution and (A) Doppler or (B) $\mathrm{CO}_{2}$ rebreathing. The dashed line represents the line of identity.

Dye-dilution measurements. Dye-dilution measurements of cardiac output were made by a technique that has been described previously in detail. ${ }^{1 t}$ In brief, a polyethylene PE-50 catheter (Clay Adams, Division of Becton Dickinson and Co., Parsippany, N.J.) was introduced percutaneously into an antecubital vein and advanced into the right atrium form delivery of indicator dye (Cardio-Green; Hynson, Westcott and Dunning, Inc., Baltimore, Md.). A 2-inch 18-gauge plastic cannula was inserted into the left brachial artery to allow continuous withdrawal of blood through an Electronics for Medicine densitometer (Honeywell Corporation, Van Nuys, Calif.). Cardiac output curves and lead II 

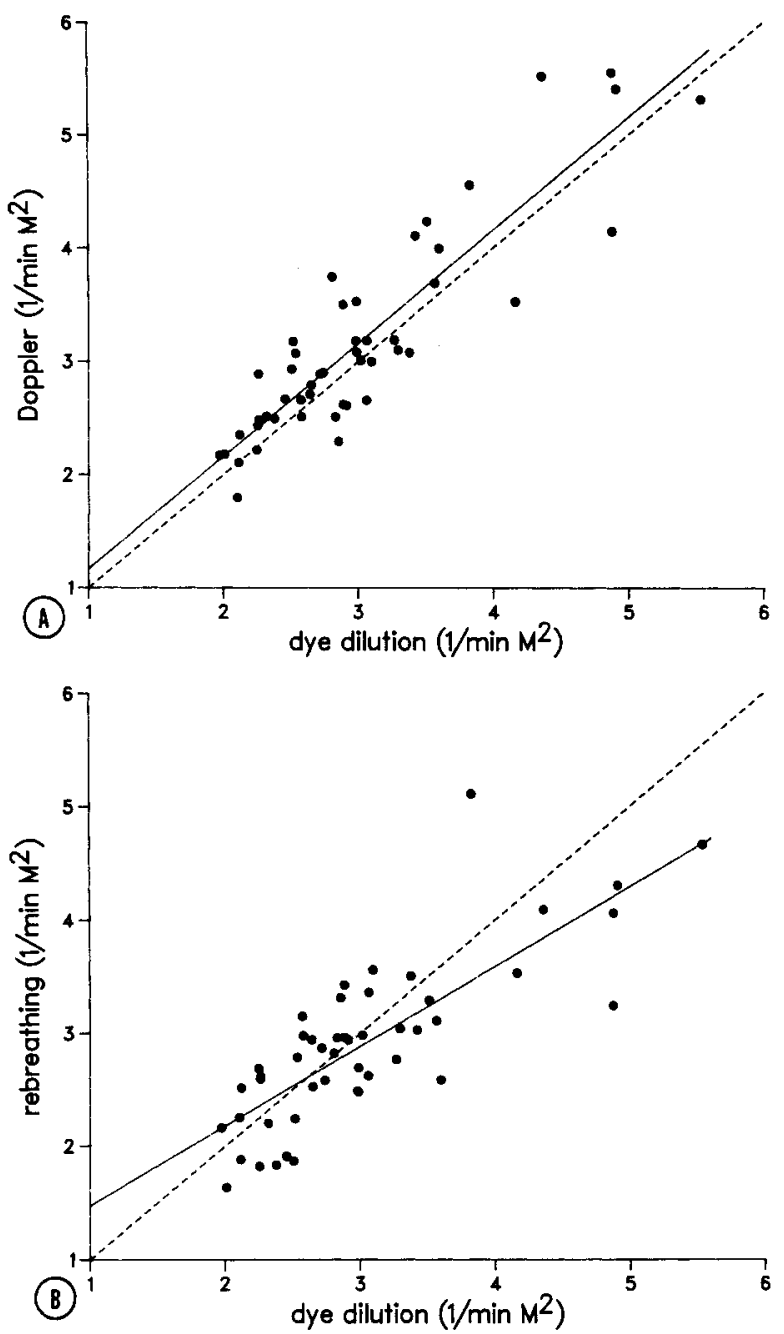

Fig. 2. Relationship between each $Q$ value measured by dye dilution and (A) Doppler or (B) $\mathrm{CO}_{2}$ rebreathing. The leastsquares regression line (solid) and line of identity (dashed) are shown.

of the ECG were recorded on the thermal recorder. Dye curves were calibrated by the addition of known quantities of dye to aliquots of blood that were drawn through the densitometer.

Data analysis. Regression and correlation analyses were used to compare $Q$ values obtained from the noninvasive Doppler and rebreathing methods, with $Q$ values obtained from the dye-dilution method for both individual and group measurements. Sample size estimates were calculated by solving (for what amounts to the smallest) $\mathrm{N}$ given the difference between the means and SDs of these differences and setting the $\alpha$ and $\beta$ errors at a specified level $(\alpha=0.05 ; \beta=0.2){ }^{12}$

\section{RESULTS}

Technically satisfactory rebreathing studies and Doppler flow tracings were obtained in all eight patients. Table $I$ lists the mean HR and $Q$ values obtained by the three methods under each condition. Strong correlations were obtained between dye-dilution and both the Doppler $(\mathrm{r}=0.99)$ and $\mathrm{CO}_{2}$ rebreathing $(\mathrm{r}=$ 0.97) methods, as illustrated in Fig. 1. The slightly weaker correlation with rebreathing was the result of significant overestimates of $\mathrm{Q}$ by this technique during head-up tilt; when the tilt values were not considered, $r=0.99$. Doppler values were, on average, slightly greater than those determined by dye dilution. In contrast, Q was somewhat underestimated in the supine position by the rebreathing method.

Fig. 2 is a graph of all 48 observations in the eight patients, plotting dye-dilution $Q$ values against $(A)$ Doppler and $(B)$ rebreathing estimates. Dye-dilution and Doppler measurements were related to the following equation: $\mathrm{Q}_{\mathrm{Dop}}=1.00 \mathrm{Q}_{\mathrm{DD}}+0.71 \mathrm{~L} / \mathrm{min} \cdot \mathrm{m}^{2}$. Although a close correlation was observed $(\mathrm{r}=$ 0.89 ), substantial differences were noted in some individual measurements, as reflected by the SEE of $0.17 \mathrm{~L} / \mathrm{min} \cdot \mathrm{m}^{2}$. Similar analysis of paired dye-dilution and rebreathing $\mathrm{Q}$ determinations resulted in the following relationship: $\mathrm{Q}_{\mathrm{CO}_{2}}=0.71 \mathrm{Q}_{\mathrm{DD}}+0.77$ $\mathrm{L} / \mathrm{min} \cdot \mathrm{m}^{2}$. The correlation with dye dilution was weaker $(\mathrm{r}=0.79)$ and the scatter was greater $(\mathrm{SEE}=$ $0.20 \mathrm{~L} / \mathrm{min} \cdot \mathrm{m}^{2}$ ) with this technique than with the Doppler method.

As illustrated in Table II, there was a close correlation between Doppler and dye-dilution values in every subject; $r$ was 0.96 or greater in six of the eight patients and averaged 0.96 . Correlation coefficients with rebreathing ranged from 0.80 to 0.97 and averaged 0.89 .

To assess the utility of Doppler echocardiography and $\mathrm{CO}_{2}$ rebreathing in clinical research, we calculated the sample sizes required for these methods to detect the changes produced by each intervention. These results are displayed in Table III. In every case, for both the dye-dilution and Doppler methods, sample sizes of six or less were sufficient to establish a change in mean Q with $95 \%$ confidence. However, only the relatively large increase resulting from isoproterenol infusion could have been established with such a small group using $\mathrm{CO}_{2}$ rebreathing. Rebreathing measurements during tilt were unreliable, and large sample sizes would have been required to demonstrate a change in response to cuff inflation or $\beta$-blockade. 
Table III. Mean Q changes in response to cuff inflation, tilt, isoproterenol, and propranolol as measured by each method, with calculated sample sizes needed to detect changes at the $5 \%$ level

\begin{tabular}{|c|c|c|c|}
\hline Intervention & $\begin{array}{l}\text { Dye dilution } \\
\left(L / \mathrm{min} \cdot \mathrm{m}^{2}\right)\end{array}$ & $\begin{array}{c}\text { Doppler } \\
\left(L / \min \cdot m^{2}\right)\end{array}$ & $\begin{array}{c}\mathrm{CO}_{2} \text { rebreathing } \\
\left(\mathrm{L} / \mathrm{min} \cdot \mathrm{m}^{2}\right)\end{array}$ \\
\hline \multicolumn{4}{|l|}{ Cuff } \\
\hline Mean $Q$ change & $-0.36^{*}$ & $-0.43^{*}$ & -0.18 \\
\hline SD & 0.22 & 0.21 & 0.42 \\
\hline Sample size & $<5$ & $<5$ & $33-40$ \\
\hline \multicolumn{4}{|l|}{ Tilt } \\
\hline Mean Q change & $-0.59 *$ & $-0.95 *$ & -0.06 \\
\hline $\mathrm{SD}$ & 0.37 & 0.36 & 0.38 \\
\hline Sample size & $<5$ & $<5$ & $>101$ \\
\hline \multicolumn{4}{|l|}{ Isoproterenol } \\
\hline Mean $Q$ change & $1.59 *$ & $1.67 *$ & $1.21 *$ \\
\hline SD & 0.64 & 0.49 & 0.71 \\
\hline Sample size & $<5$ & $<5$ & $<5$ \\
\hline \multicolumn{4}{|l|}{ Propranolol } \\
\hline Mean $Q$ change & $-0.45^{*}$ & $-0.39 *$ & -0.22 \\
\hline SD & 0.29 & 0.22 & 0.42 \\
\hline Sample size & 5 & $<5$ & $22-27$ \\
\hline
\end{tabular}

Estimated sample sizes are based on each technique's mean change and SD, with $\alpha$ set at 0.05 and $\beta$ set at 0.2 .

$* P<0.005$.

\section{DISCUSSION}

$\mathrm{Q}$ can be measured accurately by invasive techniques such as indicator dilution or thermodilution. Since these methods are associated with some discomfort and morbidity, however, their usefulness in experimental medicine has been limited to laboratory studies in relatively small numbers of patients. In view of the importance of $\mathrm{Q}$ assessment in studies of human physiology and clinical pharmacology, a reliable noninvasive method of measuring this parameter would be a valuable investigational tool.

This study was designed to compare the accuracy of $\mathrm{CO}_{2}$ rebreathing and Doppler ultrasonography in assessing acute hemodynamic changes in human laboratory subjects. As illustrated in Table I, the physiologic and pharmacologic interventions we employed resulted in a wide range of hemodynamic alterations. Infusion of isoproterenol and propranolol resulted in changes in $Q$ that paralleled those in HR, head-up tilt induced an increase in HR but a fall in $\mathrm{Q}$, and thigh cuff inflation produced in a decrease in $\mathrm{Q}$ with a minimal alteration of HR.

Our results demonstrate that close correlations between indicator dilution and noninvasive measurements of $\mathrm{Q}$ can be attained. Comparison of dye-dilution and Doppler determinations for all 48 measurements resulted in a correlation coefficient of 0.89 . These data are comparable to the published results of other series measuring SV or $\mathrm{Q}$ from the suprasternal notch, including those by Huntsman et $\mathrm{al}^{3}(\mathrm{r}=0.94 ; \mathrm{n}=110$ in 45 patients), Rose et $\mathrm{al}^{8}(\mathrm{r}=0.92 ; \mathrm{n}=16)$, and Goldberg et al. ${ }^{2}(r=0.91 ; n=16)$. Results of previous studies designed to assess the reliability of $\mathrm{CO}_{2}$ rebreathing in measuring resting $Q$ are variable. Many authors have reported close agreement with direct Fick or dye-dilution measurements during exercise but poor correlations at rest. ${ }^{13-16}$ More recently, especially with the use of the Collier equilibration technique for the estimation of mixed venous $\mathrm{PCO}_{2}$, other investigators have obtained accurate results in resting, supine patients. ${ }^{17,18}$ Our correlation coefficient of 0.79 was similar to results noted previously in our laboratory. ${ }^{19}$

When mean $Q$ values were calculated for each condition in our study, close agreement was observed between dye dilution and both noninvasive techniques. However, rebreathing measurements made in the tilt position were considerably higher than expected, probably because of alterations of the physiologic dead space and ventilation-perfusion relationships produced by postural changes. ${ }^{20.21}$

In theory, Doppler ultrasonography should be particularly well suited to evaluating changes in $\mathrm{Q}$ in individual patients. The measurement of aortic CSA is a source of significant error in the calculation of absolute 
values of SV or Q. Studies in humans indicate that this area is dependent on intra-aortic pressure and changes by $5 \%$ to $15 \%$ during the cardiac cycle. ${ }^{22,23}$ Moreover, small errors in measurement of the aortic diameter are compounded when this dimension is squared in the calculation of area. If one assumes that the dimensions of the aorta remain constant during the period of observation, relative changes in $Q$ can be assessed without consideration of the CSA. In our study group a strong correlation was found between dye dilution and Doppler determinations of $Q$ in each of the eight subjects, with a mean $r$ value of 0.96 . The expected directional changes in $Q$ were confirmed in every case. Thus even though errors in CSA measurement may have resulted in significant overestimation or underestimation of $Q$ in some subjects, relative changes in $\mathrm{Q}$ were determined with great accuracy.

The results with $\mathrm{CO}_{2}$ rebreathing were less satisfactory. Although relative changes in response to cuff inflation, isoproterenol, and propranolol were accurately quantitated by this method for the group as a whole, significant error was often observed in individual measurements.

The principal objective of this study was to assess the utility of different methods of Q measurement in clinical research. Since noninvasive techniques involve less risk and discomfort than dye dilution or Fick measurements, they generally meet with greater acceptance by human subjects. To be useful in experimental work, however, these methods must be capable of demonstrating, with statistical confidence, changes in $Q$ in a small group of patients. In our experience, comparable sample sizes were required for the Doppler and dyedilution methods to establish $\mathrm{Q}$ alterations in response to physiologic and pharmacologic maneuvers. With $\mathrm{CO}_{2}$ rebreathing, however, larger groups were required to prove a change with three of the four interventions.

Several features of our methodology and study population affect the applicability of these results. Our measurements were performed in healthy, cooperative young men at rest, and factors influencing the ease of Doppler measurements in other subjects might adversely affect the precision of $Q$ determinations. Doppler estimates of aortic flow are technically difficult in moving patients, for example, and for this reason $\mathrm{CO}_{2}$ rebreathing may be a more accurate method of assessing the response of $\mathrm{Q}$ to exercise. In addition, we cannot exclude the possibility that propranolol and isoproterenol caused regional changes in the ventilation/perfusion ratio, resulting in rebreathing measurement errors that would not be seen with agents having no effect on bronchiolar tone. However, it should be noted that rebreathing measurements of responses to propranolol and isoproterenol were no less accurate than those of changes produced by thigh cuff inflation.

In summary, pulsed Doppler echocardiography and $\mathrm{CO}_{2}$ rebreathing are useful techniques for the noninvasive measurement of $\mathrm{Q}$. However, calculation of $\mathrm{ab}$ solute values for $\mathrm{Q}$ by the Doppler method necessitates accurate measurement of the aortic cross-sectional area. Since this potential source of error does not complicate comparisons of serial measurements of $\mathrm{Q}$ in individual subjects, the Doppler method is particularly well suited to measuring acute $\mathrm{Q}$ changes. $\mathrm{CO}_{2}$ rebreathing can provide reasonably accurate estimates of resting $\mathrm{Q}$ in healthy subjects. In our laboratory, however, measurements of changes in $\mathrm{Q}$ in individual patients are less reliable. These findings suggest that both noninvasive methods may be useful in human hemodynamic studies, especially when applied to a group of subjects. However, Doppler echocardiography is a much more accurate means of quantifying $Q$ responses to pharmacologic and physiologic maneuvers.

\section{References}

1. Chandraratna PAN, Nanna M, McKay C, Nimalasurlya A, Swinney A, Elkayam U, Rahintoola SH. Determination of cardiac output by transcutaneous continuouswave ultrasonic Doppler computer. J Am Coll Cardiol 1984;53:234-7.

2. Goldberg SJ, Sahn DJ, Allen HD, Valdes-Cruz LM, Hoenecke $\mathrm{H}$, Carnahan Y. Evaluation of pulmonary and systemic blood flow by two-dimensional Doppler echocardiography using fast fourier transform spectral analysis. Am J Cardiol 1982;50:1394-1400.

3. Huntsman LL, Stewart DK, Barnes SR, Franklin SB, Colocousis JS, Hessel EA. Noninvasive Doppler determination of cardiac output in man: clinical validation. Circulation 1983;67:593-602.

4. Lewis JF, Kuo LC, Nelson JG, Limacher MC, Quinones MA. Pulsed Doppler echocardiographic determination of stroke volume and cardiac output: clinical validation of two new methods using the apical window. Circulation 1984;70:425-31.

5. Ihlen H, Amlie JP, Dale J, et al. Determination of cardiac output by Doppler echocardiography. Br Heart J 1984; 51:54-60.

6. Distante A, Moscarelli E, Rovai D, L'Abbate A. Monitoring of changes in cardiac output by transcutaneous aortovelography, a non-invasive Doppler technique: comparison with thermodilution. J Nucl Med Allied Sci 1980;24:171-5.

7. Elkayam U, Gardin JM, Berkley R, Hughes CA, Henry WL. The use of Doppler flow velocity measurement to 
assess the hemodynamic response to vasodilators in patients with heart failure. Circulation 1983;67:377-83.

8. Rose JS, Nanna M, Rahintoola SH, Elkayam U, McKay C, Chandraratna PAN. Accuracy of determination of changes in cardiac output by transcutaneous continuous wave Doppler computer. J Am Coll Cardiol 1984;54:1099-1102.

9. Collier CR, Affeldt JE, Farr AF. Continuous rapid infrared $\mathrm{CO}_{2}$ analysis. J Lab Clin Med 1955;45:526-39.

10. Comroe JH. Physiology of respiration: an introductory text, ed 2. Chicago: Year Book Medical Publishers, Inc, 1974.

11. Julius S, Amery A, Whitlock LS, Conway J. Influence of age on the hemodynamic response to exercise. Circulation 1967;36:222-30.

12. Beyer WH, ed. Handbook of tables for probability and statistics, ed 2. Boca Raton, FL: CRC Press Inc, 1968:286-9.

13. Clausen JP, Larsen OA, Trap-Jensen J. Cardiac output in middle-aged patients determined with $\mathrm{CO}_{2}$ rebreathing method. J Appl Physiol 1970;28:337-42.

14. Ferguson RJ, Faulkner JA, Julius S, Conway J. Comparison of cardiac output determined by $\mathrm{CO}_{2}$ rebreathing and dye-dilution methods. J Appl Physiol 1968;25: 450-4.

15. Reybrouck T, Amery A, Billiet L, Fagard R, Stijns H. Comparison of cardiac output determined by a carbon dioxide rebreathing and direct Fick method at rest and during exercise. Clin Sci Mol Med 1978;55:445-52.
16. Zeidifard E, Sliverman M, Godfrey S. Reproducibility of the indirect $\left(\mathrm{CO}_{2}\right)$ Fick method for calculation of cardiac output. J Appl Physiol 1972;33:141-3.

17. Franciosa JA, Ragan DO, Rubenstone SJ. Validation of the $\mathrm{CO}_{2}$ rebreathing method for measuring cardiac output in patients with hypertension or heart failure. J Lab Clin Med 1976;88:672-82.

18. Muiesan G, Sorbini CA, Solinas E, Grassi V, Casucci G, Petz E. Comparison of $\mathrm{CO}_{2}$ rebreathing and direct Fick methods for determining cardiac output. J Appl Physiol 1968;24:424-9.

19. Colfer HT, Cottier C, Sanchez R, Julius S. Role of cardiac factors in the initial hypotensive action of beta-adrenergic blocking agents. Hypertension 1984;6: 145-51.

20. Larson CP, Severinghaus JW. Postural gradients in dead space and $\mathrm{CO}_{2}$ gradients breathing air and $\mathrm{O}_{2} . \mathrm{J}$ Appl Physiol 1962;17:417-20.

21. West JB. Regional differences in gas exchange in the lung of erect man. J Appl Physiol 1962;17:893-8.

22. Greenfield JC, Patel DJ. Relation between pressure and diameter in the ascending aorta of man. Circ Res 1962;10:778-81.

23. Merillon JP, Motte G, Fruhaud J, Masquet C, Gourgon $\mathrm{R}$. Evaluation of the elasticity and characteristic impedance of the ascending aorta in man. Cardiovasc Res 1978;12:401-6. 